\title{
Las adolescentes indígenas: Una prioridad de hoy para un mejor futuro nacional en el 2015
}

Population Council

Follow this and additional works at: https://knowledgecommons.popcouncil.org/departments_sbsr-pgy

Part of the Demography, Population, and Ecology Commons, Education Commons, Family, Life Course, and Society Commons, Indigenous Studies Commons, and the International Public Health Commons How does access to this work benefit you? Let us know!

\section{Recommended Citation}

"Las adolescentes indígenas: Una prioridad de hoy para un mejor futuro nacional en el 2015." Guatemala: Population Council, 2015. 


\section{Las}

\section{adolescentes indígenas}

\section{Una prioridad de hoy para un mejor futuro nacional en el 2015}

\section{La educación secundaria}

Debido a diversos factores, como la pobreza, el matrimonio a temprana edad y el poco acceso a las escuelas, las adolescentes indígenas no se inscribren o no terminan sus estudios de secundaria.

\section{Situación actual}

\section{Tasas de inscripción ${ }^{1}$ muy bajas}

La tasa de inscripción de adolescentes indígenas guatemaltecas en secundaria es la más baja de Centroamérica. Estudios recientes indican que una de cada diez se inscribe en la escuela secundaria ${ }^{2}$ y una de cada cien adolescentes indígenas que vive en pobreza extrema se inscribe en la secundaria. ${ }^{3}$

\begin{tabular}{|c|c|}
\hline $\begin{array}{ll}\text { El Salvador } & 61 \% \\
\text { Nicaragua } & 59 \%\end{array}$ & $\begin{array}{ll}\text { Honduras } & 48 \% \\
\text { Guatemala } & 33 \%\end{array}$ \\
\hline
\end{tabular}

\section{No hay programas de nivelación}

Uno de los problemas que enfrentan las adolescentes indígenas es que no existen programas de nivelación de educación primaria y secundaria, razón por la cual, cuatro de cada diez adolescentes, entre 13 a 15 años, están en la primaria. ${ }^{4}$

Otro de los efectos de la falta de nivelación, es el atraso en los estudios en relación a la edad. En ese sentido, ocho de cada diez estudiantes indígenas que se inscriben en la secundaria tienen dos o más años de atraso con respecto a su edad. ${ }^{5}$

\begin{tabular}{|c|}
\hline $\begin{array}{l}\text { De cada diez niñas y adolescentes indígenas } \\
\text { en Guatemala: }\end{array}$ \\
\hline $\begin{array}{l}\text { ocho están en la escuela primaria a los } 12 \text { años; } \\
\text { cinco están en la escuela primaria a los } 14 \text { años; y } \\
\text { dos están en la escuela primaria a los } 16 \text { años. }\end{array}$ \\
\hline
\end{tabular}

\section{El matrimonio en adolescentes indígenas} se asocia con abandono ${ }^{6}$ escolar

El matrimonio es otro de los factores que no permite que las adolescentes indígenas continúen sus estudios. Las cifras muestran que una de cada tres adolescentes indígenas, de 15 a 19 años, están casadas y solo tres de cada cien casadas asisten a la secundaria. ${ }^{7}$

\section{Cobertura escolar es desigual por nivel de ingresos, étnia y área geográfica}

Otro de los problemas que enfrentan las adolescentes indígenas es que sólo dos de cada diez escuelas secundarias públicas están ubicadas en áreas rurales. ${ }^{8}$
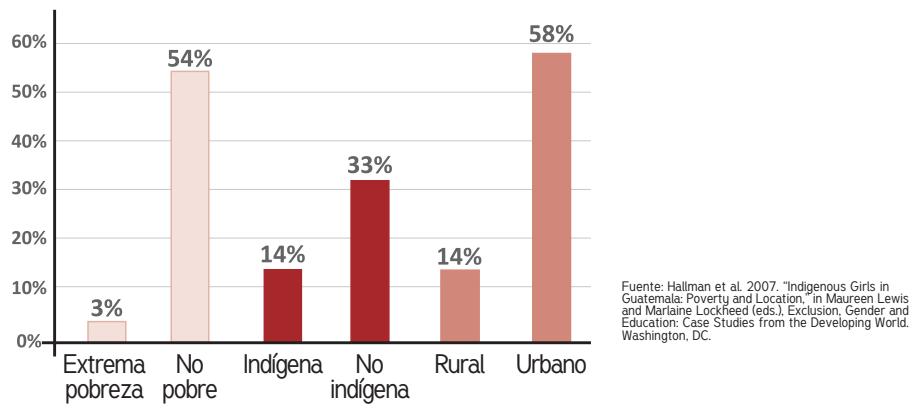

\section{El subsidio público en el nivel secundario}

De la primaria a la secundaria los gastos directos de la educación aumentan cinco veces para las escuelas públicas y dieciséis veces para programas como el PRONADE o las cooperativas secundarias. ${ }^{9}$

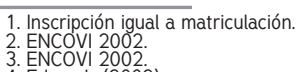




\title{
Acciones para lograr un mejor futuro nacional:
}

\section{Aumentar el subsidio público en el nivel secundario}

Expandir programas de becas ${ }^{10} \mathrm{y} / \mathrm{o}$ transferencias condicionadas es importante para apoyar la retención escolar de adolescentes y mujeres jóvenes. Estas deben cubrir los gastos directos de la secundaria y enfocarse en las estudiantes más necesitadas. Asimismo, deben condicionar que completen cada ciclo educativo.

\section{Incrementar la inscripción en secundaria}

Aumentar el número de escuelas secundarias y mejorar la infraestructura existente en las áreas rurales es necesario para optimizar la transición de las adolescentes de la primaria a la secundaria. Además, se debe incrementar el número de docentes bilingües e implementar programas de escuelas secundarias en escuelas primarias. Estas acciones permitirán la expansión de la cobertura de la educación secundaria, que debe asegurar a las nuevas generaciones el desarrollo de capacidades y herramientas para lograr la sostenibilidad de procesos para una vida digna.

\section{Aumentar la completación ${ }^{11}$ del nivel secundaria}

Fortalecer y expandir programas alternativos para secundaria, adaptados para responder a un modelo de educación bilingüe y multicultural en zonas rurales, ${ }^{12}$ es imprescindible para que las adolescentes completen la educación secundaria.

\section{Ampliar o establecer programas de nivelación}

Mejorar las tasas de inscripción en secundaria, con programas que faciliten la transición de la primaria a la secundaria. Estos deben poner énfasis en captar y retener a estudiantes que abandonan la primaria.

\section{Mejorar la calidad educativa de la secundaria}

Apoyar la implementación y aplicación de los nuevos currículos nacionales actualizados con pertinencia cultural y procesos de monitoreo y evaluación dentro de los ejes de equidad y género, permitirán mejorar la calidad educativa de la secundaria. Se debe promover el currículo nacional básico, desarrollar materiales pertinentes, establecer estándares de competencia, profesionalizar a docentes y mejorar el nivel salarial del magisterio. ${ }^{13}$

\section{Expandir programas de educación técnica vocacional y habilidades para la vida}

Ofrecer capacitaciones sobre habilidades vocacionales para adolescentes y jóvenes dentro y fuera del sistema educativo secundario permite fortalecer sus habilidades técnicas y para la vida. Estas capacitaciones deben ser prácticas y relevantes para el contexto rural, con enfoque en iniciativas emprendedoras dentro de la comunidad.

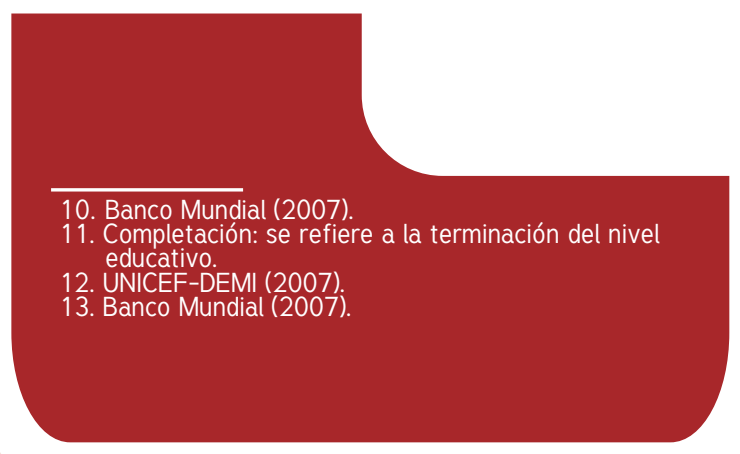

\section{1) Population Council}

\author{
Bibliografía \\ 1. ENCOVI, 2002 \\ 2. Hallman et al. 2007. "Indigenous Girls in Guatemala: Poverty and Location," \\ in Maureen Lewis and Marlaine Lockheed (eds.), Exclusion, Gender and" \\ Education: Case Studies from the Developing World. Washington, DC \\ 3. Edwards, Education and Poverty in Guatemala. GUAPA 2002.
}




\section{Las \\ adolescentes indígenas}

\section{Una prioridad de hoy para un mejor futuro nacional en el 2015}

\section{La educación primaria}

\section{Esfuerzos para expandir la educación bilingüe en áreas rurales, han \\ contribuido a incrementar la tasa de asistencia a la escuela primaria. ${ }^{1}$ Sin embargo, para las niñas y \\ adolescentes indígenas, no inscribirse o repetir el grado es una constante que incrementa el riesgo de no completar la escuela.}

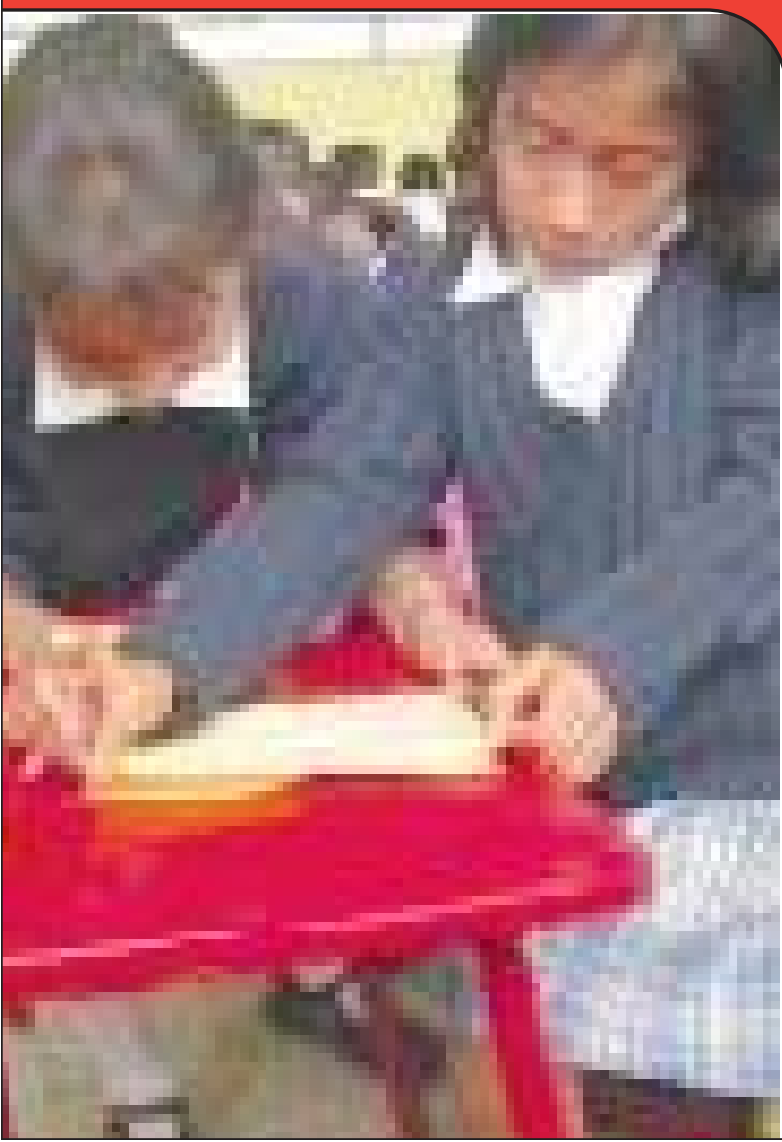

\section{Situación actual}

\section{Inscripción neta2 ha aumentado}

Los esfuerzos por expandir la educación bilingüe en áreas rurales permitió que un 31.3 por ciento de las niñas completaran de primero a tercer año en tres años. Actualmente, el promedio de escolaridad en población indígena es de 2.0 años. ${ }^{3}$

\begin{tabular}{|c|c|c|}
\hline \multicolumn{3}{|c|}{$\begin{array}{l}\text { Mujeres jóvenes ( } 15 \text { a } 24 \text { ) con primaria comp } \\
\text { o más años de escolaridad }\end{array}$} \\
\hline 2002 & Indígenas $11 \%$ & \\
\hline 2006 & Indígenas $25 \%$ & No indígenas \\
\hline
\end{tabular}

\section{Tasa de completación ${ }^{4}$ primaria es muy baja}

Un alto porcentaje de niñas indígenas no completa su educación primaria. Las cifras indican que solo dos de cada cuarenta completan sus estudios primarios (5\%). ${ }^{5}$

En ese sentido, dos de cada diez niñas indígenas completan la primaria a tiempo, a diferencia de seis de cada diez niñas no indígenas. ${ }^{6}$

\section{Carencia de recursos económicos impide la asistencia}

Uno de los problemas que enfrentan las niñas indígenas es la falta de recursos económicos para continuar sus estudios. Dentro de este marco, cuatro de cada diez niñas indígenas no inscritas en la primaria reportaron no asistir por falta de dinero para transporte, materiales y uniformes, entre otros. ${ }^{7-8}$

\section{Repitencia9 escolar limita la permanencia ${ }^{10}$}

Repetir el grado es una constante que se produce en las niñas indígenas que viven en áreas rurales. Cuatro de cada diez presentan rezago al menos de dos grados escolares o más por debajo del que le corresponde de acuerdo a su edad. ${ }^{11}$

\section{Efecto de la étnia, género y lugar de residencia}

Factores, como la falta de dinero producen que las niñas indígenas en pobreza extrema tengan mayor riesgo de no inscribirse en la escuela primaria, de inscribirse tarde o de no completar la escuela. ${ }^{2}$

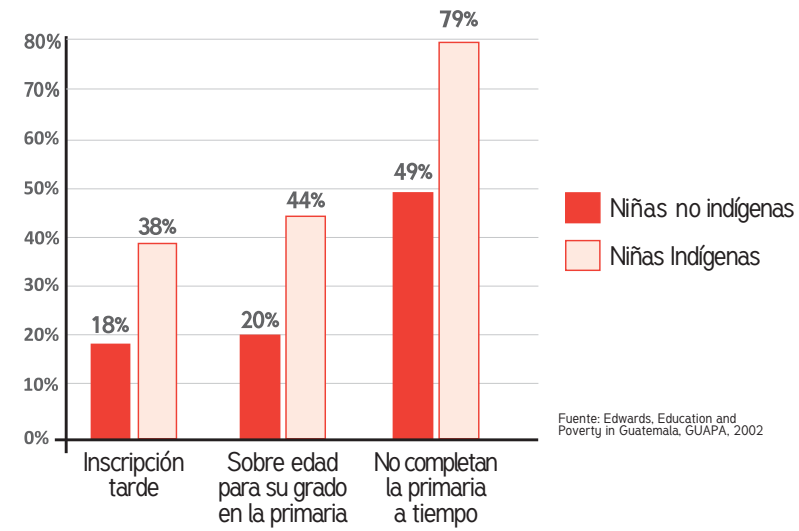

\section{Gasto público en educación}

En 2005, Guatemala fue uno de los paises de la región que asignó a educación, menos porcentaje del Producto Interno Bruto (PIB), equivalente a un 2.5 por ciento. Este factor factor, en una de las principales limitaciones para dar un salto cuantitativo de mayor alcance en materia educativa. ${ }^{12}$

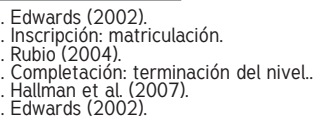

Mas y mejor educación en Guatemala (2007).

Repitencia: cuando se pierde un grado y hay que repetirlo. 10. Permanencia: retención en el sistema educativo. 1. Edwards (200 


\section{Acciones para lograr un mejor futuro nacional:}

\section{Aumentar el gasto público en educación}

Aumentar la asignación del Producto Interno Bruto (PIB) como mínimo a un 4.3 por ciento es una de las acciones que permitirá mejorar la situación y condición de la educación primaria de las niñas indígenas rurales. ${ }^{13}$ Además, es necesario apoyar la política transversal de educación 2008-2012,14 con un aumento en la inversión educativa por medio de la reasignación de recursos y recursos adicionales (movilizar nuevos recursos y/o reorientar el gasto). ${ }^{15}$

\section{Inscripción a tiempo en la escuela preprimaria y primaria}

Sensibilizar y organizar a la comunidad es necesario para que los padres y madres insciriban oportunamente a sus hijas en la escuela preprimaria y primaria. $^{16}$

\section{Aumentar la retención escolar y mejorar la calidad educativa} Incrementar la contratación de maestros y maestras bilingües en el área rural, principalmente para el nivel pre-primario y los primeros años de la primaria, es impresicindible para incrementar la retención escolar y mejorar la calidad educativa de las niñas indígenas. $^{17}$

Para el efecto, se debe revisar el sistema de incentivos dirigido al magisterio, con el fin de ubicar a los más capaces en las escuelas que lo requieran.

Asimismo, se debe implementar el eje de diversidadetnica en el currículo educativo, el cual debe ser actualizado de acuerdo a la pertinencia cultural y debe ser monitoreado y evaluado al momento de su implementación.

\section{Priorizar las comunidades indígenas}

Ampliar la cobertura en preprimaria y primaria, ${ }^{18}$ por medio de la expansión del modelo actual de Educación Bilingüe intercultural (EBI) es importante para reducir repetición y deserción escolar entre estudiantes indígenas.

También se debe elevar la conciencia social de la EBI, como un derecho humano y bien público de la educación.

\section{Aumentar la cobertura de becas y del programa de transferencias condicionadas}

Promover la ampliación de los programas de transferencias condicionadas y el monitoreo y evaluación de las mismas para determinar el efecto en las niñas indígenas, es una de las formas de mejorar la educación primaria para este sector de la población guatemalteca. Se debe ampliar el programa de becas para niñas indígenas y refacción escolar universal para preprimaria y primaria.

\section{Establecer programas de} nivelación para estudiantes y disminuir la tasa de sobre-edad

Ampliar los modelos y/o programas existentes de nivelación para estudiantes en el área rural de Guatemala es una forma en que se puede disminuir la tasa de sobre edad, es decir, del retraso en grado escolar que las niñas tienen con respecto a su edad. De igual forma, es necesario apoyar las intervenciones de inscripción a tiempo y educación más efectiva para disminuir la reprobación y la deserción escolar.

\section{(1) Population Council}

\section{Bibliografía}

1. Edwards. Education and Poverty in Guatemala, GUAPA, 2002. 2. Hallman et al. 2007. "Indigenous Girls in Guatemala: Poverty and Location," in Maureen Lewis and Marlaine Lockheed (eds.), Exclusion, Gender and Education: Case Studies from the Developing World. Washington, DC

3. Informe Nacional de Desarrollo Humano. Guatemala 2005. PNUD

4. Banco Mundial, Proyecto para ampliar la cobertura y calidad de la educación secundaria, Documento de Evaluación, 2007.

5. UNICEF-DEMI. Situación de la niña indígena en Guatemala. 2007.

6. Políticas Educativas 2008-2012. MINEDUC, Guatemala 2008.

7. Más y mejor educación en Guatemala (2008-2021). Instituto

Centroamericano de Estudios Fiscales -ICEFI. Julio 2007.

8. Rubio, Fernando (2004). “Educación bilingüe en Guatemala: situación y desafíos". Banco Mundial. 


\section{Las \\ adolescentes indígenas}

\section{Una prioridad de hoy para un mejor futuro nacional en el 2015}

\section{La salud \\ reproductiva}

\section{Los embarazos en las adolescentes indígenas producen que sus \\ expectativas de vida se relacionen \\ al cuidado del hogar y que no \\ existan posibilidades de culminar \\ la escuela primaria, secundaria o realicen un oficio o profesión.}

\section{Situación actual}

\section{Alta tasa de embarazo entre mujeres jóvenes indígenas}

Más de la mitad de las mujeres jóvenes indígenas del área rural han tenido un parto antes de los 20 años ${ }^{1}$ y siete de cada diez sin educación primaria tuvieron un parto antes de los 20 años. ${ }^{1}$

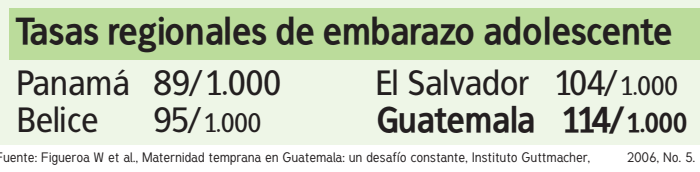

\section{Bajo uso de anticonceptivos modernos}

Según datos proporcionados por una investigación realizada por el Instituto Guttmacher en Guatemala, la gran mayoría de las adolescentes de 15 a 19 años, que son sexualmente activas no desean tener un hijo pronto. Sin embargo, la proporción que usa un método moderno es tres veces mayor entre las adolescentes no indígenas que las indígenas, es decir, de un 26 por ciento a un 8 por ciento.

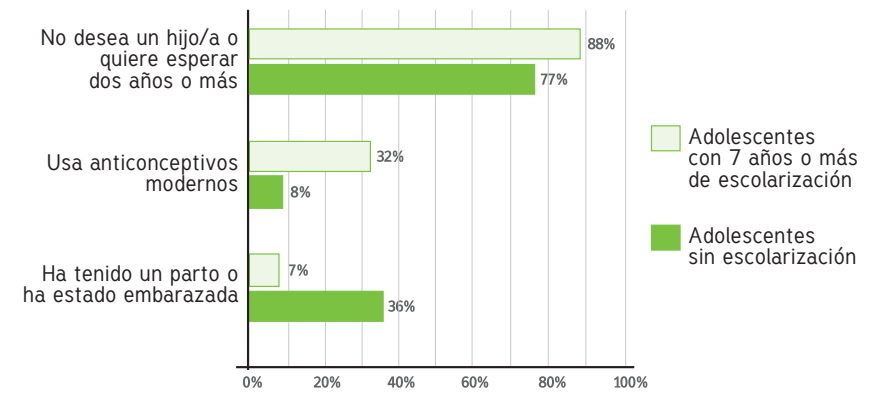

\section{Maternidad temprana y autonomía}

La maternidad temprana reduce la autonomía social y económica de la mujer, pone en riesgo su vida y la de su bebé. Las madres jóvenes suelen perpetuar un ciclo de pobreza.

\section{Deficiente salud reproductiva entre mujeres indígenas rurales}

Las bajas tasas de atención prenatal, del parto y el estado de salud de las mujeres pobres contribuyen a la razón de mortalidad materna que es de 153 por 100 mil nacidos vivos y la mortalidad infantil es de 39 por cada 100 mil nacidos. Estas tasas son las más altas en la región. La mortalidad materna entre mujeres indígenas es tres veces más alta que la tasa de mujeres no indígenas. Esto significa que la tasa de mortalidad en mujeres indígenas es de 211 muertes por cada 100 mil nacidos vivos, mientras las mujeres no indígenas tienen una tasa de 70 por 100 mil nacidos vivas.

Otro dato preocupante es que alrededor del 18 por ciento de las muertes en este segmento de la población ocurren en adolescentes.

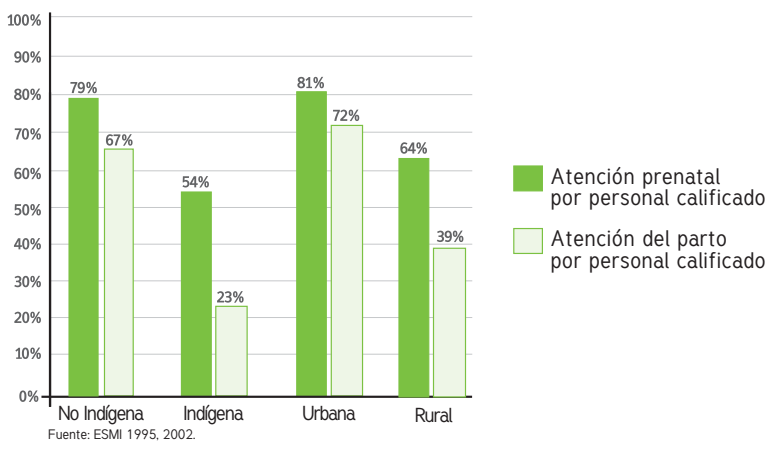

\section{Violencia basada en género}

La desigualdad entre el hombre y la mujer en términos de nivel de educación y poder adquisitivo hace que las expectativas sociales de la mujer permanezcan bajas y que el abuso físico, verbal y sexual continúe siendo tolerado y perpetuado. 


\section{Acciones para lograr un mejor futuro nacional:}

\section{Reducir el embarazo entre adolescentes}

Promover la permanencia (retención) escolar del nivel primario y secundario es una de las formas para reducir el embarazo en adolescentes, ya que la brecha más grande está relacionada con la falta educación. La proporción de mujeres que dio a luz siendo adolescente es tres veces mayor entre las mujeres sin educación, que entre aquellas que tenían por lo menos seis años o más de escolaridad. ${ }^{2}$

2. Aumentar el acceso a la atención prenatal, en el parto y post-natales por personal capacitado

Garantizar servicios de calidad y diferenciados para las adolescentes permitirá identificar riesgos y atención de complicaciones obstétricas en forma oportuna y asegurarles un embarazo y un parto seguros.

\section{Aumentar el acceso a métodos anticonceptivos, consejería informada y provisión}

Incrementar los puntos de acceso del sector público por medio de atención integral a la niñez y a la mujer y garantizar la disponibilidad e información de anticonceptivos en el área rural tomando en cuenta la pertinencia cultural y la confiabilidad de los servicios.

\section{Expandir acceso a información sobre} salud reproductiva en la escuela primaria y secundaria

Una de las estrategias que permite el incremento de los conocimientos en las juventudes es implementar programas que brinden información sobre la salud reproductiva en los últimos grados de la escuela primaria y en los primeros grados del básico. Estos deben ser apropiados a la edad, experiencia y pertinencia cultural, tanto para maestros como para las mujeres adolescentes indígenas. Asimismo, se debe incluir en la currícula de formación contenidos sobre: derechos y responsabilidades para la promoción y auto cuidado de la salud, sexualidad, el embarazo precoz y no planeado, factores de riesgo que contribuyen y afectan la morbi-mortalidad materno infantil. ${ }^{5}$
5. Expandir programas educativos de salud reproductiva integrales fuera de la escuela

Implementar estrategias efectivas para abrir espacios fuera de la escuela dirigidos a adolescentes es necesario para atender a las que están en riesgo de embarazo temprano y otros problemas de salud reproductiva. Dichas estrategias deben involucrar a los principales tomadores de decisiones de sus hogares, ya sean sus padres, madres, suegras y parejas.

6. Expandir espacios amigables de atención integral para la mujer en las áreas rurales

Promover Espacios Amigables es necesario para informar a los y las adolescentes sobre el derecho de la salud, los derechos sexuales y reproductivos, así como para proporcionar educación en salud e intercambiar y compartir experiencias con personal de su misma edad. Estos servicios han sido una excelente estrategia para informar a adolescentes, los cuales deben ser accesibles en áreas rurales, con pertinencia cultural.

7. Incluir herramientas para la vida Los programas escolares dentro y fuera de la escuela deben incluir información relevante para que las juventudes que viven en situación de pobreza en áreas rurales puedan realizar sus planes de vida, tomar decisiones asertivas, experimentar roles de género equitativos, fortalecer su autoestima y exponer a las niñas y niños a modelos alternativos de vida.

\section{Reducir violencia basada en género}

Es necesario promover el cumplimiento de las leyes contra la violencia de género para que ésta sea vista como un problema de todos y todas las guatemaltecas, donde la práctica del sistema judicial sea eficiente y oportuna. En ese sentido, se debe sensibilizar y capacitar a diversos actores de la sociedad civil, como líderes comunitarios, promotores comunitarios para que identifiquen los casos y hagan la referencia oportuna. Para minimizar la violencia basada en género, también, es necesario impulsar programas de educación formal y no formal para adolescentes, con el fin de que conozcan y hagan valer sus derechos a la salud y sus derechos sexuales y reproductivos, así como promover nuevos roles de género desde las edades tempranas para aumentar las opciones educativas y vocacionales, que permitan a las mujeres generar ingresos económicos propios. ${ }^{2}$
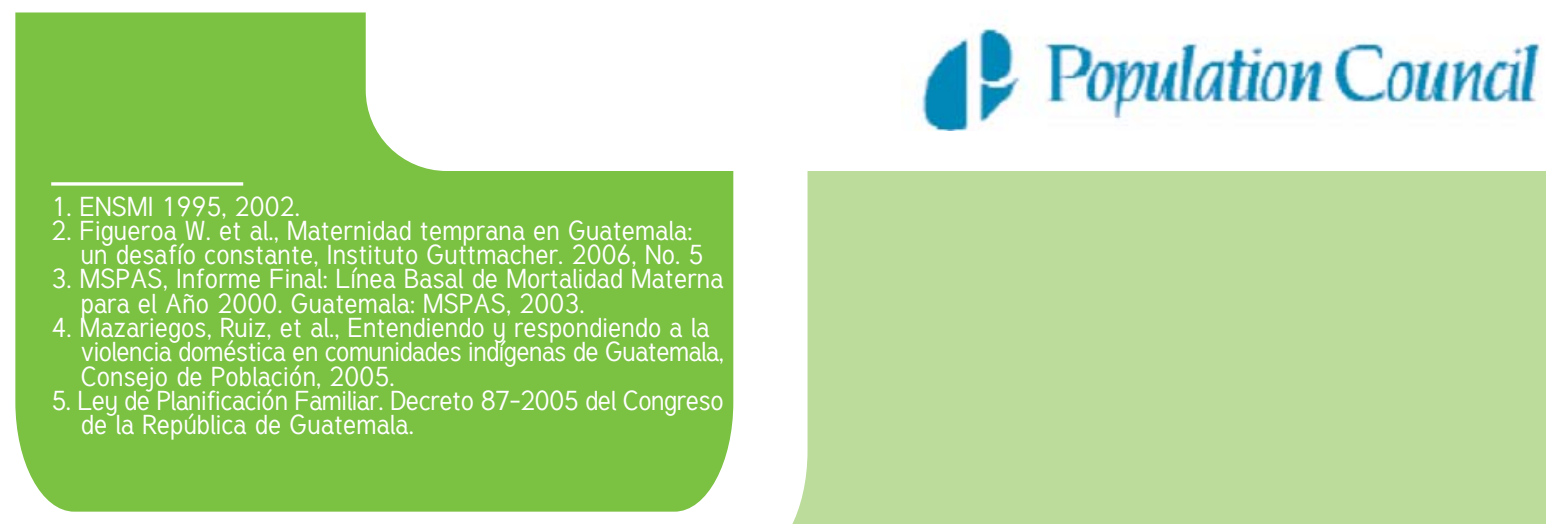
Las

adolescentes indígenas

\section{Una prioridad de hoy para un mejor futuro nacional en el 2015}

\section{Medios de vida}

Actualmente, un 53 por ciento de adolescentes, entre 10 y 14 años se incorporan al trabajo infantil.1 Pero, la situación más difícil es para las adolescentes indígenas, que no asisten a la escuela y no trabajan a cambio de un pago. ${ }^{2}$

\section{Situación actual}

Existen diversos factores que provocan que las niñas y las adolescentes trabajen, entre ellos se encuentran el abandono o muerte del padre, quien es el que en la mayoría de casos da el aporte económico al hogar. Estas situaciones hacen que la madre y sus hijos e hijas tengan que trabajar para llevar el sustento a sus hogares.

También existen otros casos, donde el papá está presente pero la situación económica no permite el desarrollo familiar, por lo que las niñas y adolescentes deben trabajar.

Jovenes que no estudian ni reciben dinero por el trabajo que realizan:

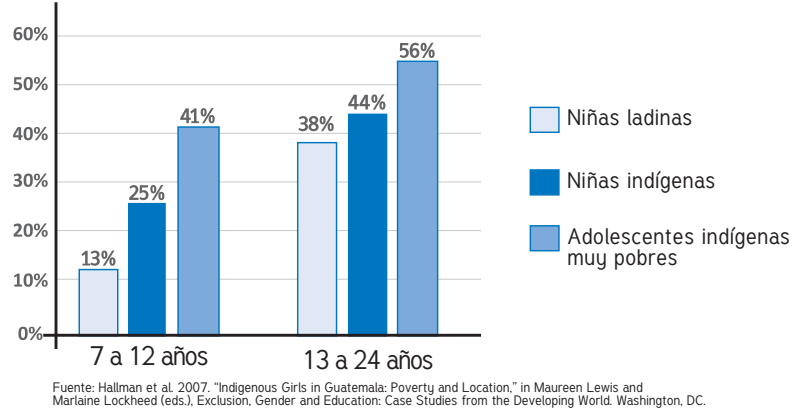

Trabajo doméstico no pagado

Otro de los problemas que enfrentan las niñas y adolescentes indígenas es que desde muy temprana edad cuidan a sus hermanos y hermanas menores y se ocupan de los oficios domésticos en la casa. Estas situaciones se deben a la extrema pobreza en que viven y a la alta tasa de fertilidad en mujeres indígenas. (4.4 para la población total y de 5.2 para el área rural). ${ }^{3}$

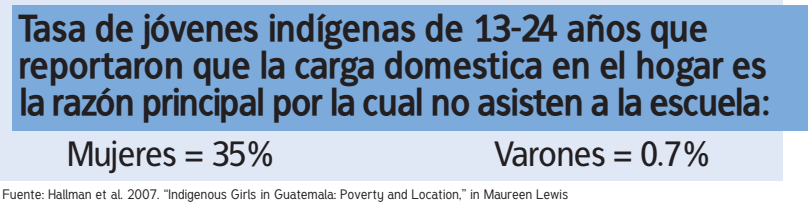

\section{Trabajo en el sector agrícola}

EL 55\% de la niñez que trabaja lo hace en el sector agrícola, con el agravante de ser un trabajo sub-pagado, que no es equitativo entre hombres y mujeres. ${ }^{1}$

\section{Trabajo en maquilas}

Tres de cada diez niñas y adolescentes trabajan en la producción de artesanías u otras manualidades y un número creciente en maquilas, donde ganan menos que las personas adultas y muchas veces trabajan horarios extensos en condiciones no saludables.

\section{Servicio doméstico en áreas urbanas}

Muchas adolescentes indígenas migran de comunidades rurales para trabajar como empleadas domésticas en casas particulares. A pesar de que reciben un salario, no se visibiliza su trabajo, ya que el trabajo doméstico no es cuantificado. Además, las adolescentes sufren, pues están lejos de sus familias y del apoyo que les dan y están en riesgo de abuso laboral, físico, emocional y/o sexual.

\section{Trabajo infantil}

La ley en Guatemala establece la edad de 14 años como el mínimo para el trabajo pagado por necesidad económica, estableciendo excepciones calificadas. No obstante, se estima que aproximadamente la mitad de los adolescentes de 10 a 14 años tienen que trabajar para contribuir económicamente a su hogar.

\section{Aspiraciones de las niñas indígenas contrastan con la realidad}

Muchas adolescentes indígenas desean adquirir capacitación profesional para ser médicas, abogadas y maestras, entre otros. Sin embargo, admiten que sería casi imposible conseguir este tipo de posición dentro de sus comunidades. El enfoque del sistema educacional no refleja la realidad de los medios de vida disponibles en las áreas rurales. 


\section{Acciones para lograr un mejor futuro nacional:}

\section{Garantizar los derechos laborales y controlar el trabajo infantil}

Establecer mecanismos eficientes en el Estado para asegurar el cumplimiento de leyes y normas vigentes de protección integral de los derechos de la niñez y adolescencia permitirá garantizar los derechos laborales y controlar el trabajo infantil. Es necesario que las leyes nacionales específicas estén en armonía con los convenios internacionales suscritos y ratificados por el país en materia de trabajo infantil. Para el efecto, se debe fortalecer a las entidades de gobierno, con el propósito que velen y monitoreen la aplicación de dicha legislación.

\section{Aumentar el acceso a la escuela y} mejorar la calidad educativa

Incrementar la inversión en educación secundaria en áreas rurales, mejorar la calidad de la enseñanza y apoyar el acceso de los y las niñas a escuelas cercanas a sus hogares permitirá sensibilizar a los padres y madres sobre la importancia de que terminen sus estudios para tener un mejor futuro. Con lo anterior, también se logrará evitar que los y las niñas solo trabajen.

Es necesario promover modelos innovadores y alternativos como la alfabetización digital y facilitar procesos de pasantías y mentoría empresariales, que tomen en cuenta la demanda del mercado laboral y las aspiraciones de las jóvenes, con el propósito de aumentar sus oportunidades laborales.

\section{Proveer herramientas para la vida} dentro y fuera del sistema educativo

Desarrollar metodologías educativas y capacitaciones prácticas sobre habilidades para la vida, contextualizadas a la región.

\section{Salarios equitativos para hombres y mujeres}

Ampliar las oportunidades de educación y capacitación vocacional de las mujeres indígenas e incidir en el sector público y privado para que los salarios sean iguales por el mismo trabajo es importante para lograr sistemas más equitativos de trabajo. Actualmente, las mujeres indígenas reciben tres veces menos del retorno económico por año de escolaridad que los hombres no indígenas.

\section{Conectar comunidades con recursos y facilitar la creación y expansión de micro-empresas}

Promover programas de desarrollo rural en los que se conecten a productores y emprendedores rurales con mercados nacionales e internacionales para que las adolescentes y jóvenes puedan tener mejores oportunidades de vida y mejorar sus condiciones económicas . En ese sentido, se deben incentivar programas de micro-finanzas y de microempresas que den capacitación y seguimiento apropiado para implementar nuevos modelos para empoderar a mujeres jóvenes en el emprendimiento de negocios o actividades productivas. Se deben priorizar las comunidades más rezagadas y asegurar que los planes para el desarrollo económico tomen en cuenta las necesidades de niñas y adolescentes indígenas.

\section{Involucrar al sector privado}

Incentivar al sector privado para invertir en la formación profesional y vocacional de las mujeres jóvenes indígenas.

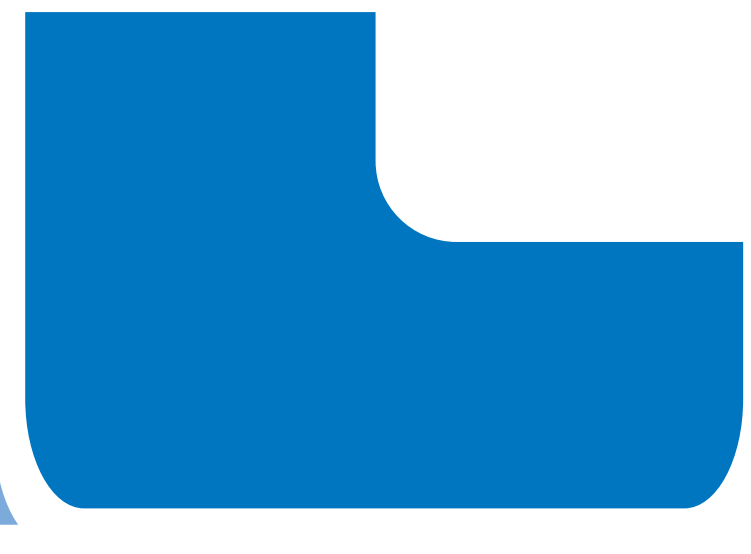

\section{(2) Population Council}


Las adolescentes indígenas

\section{Una prioridad de hoy para un mejor futuro nacional en el 2015}

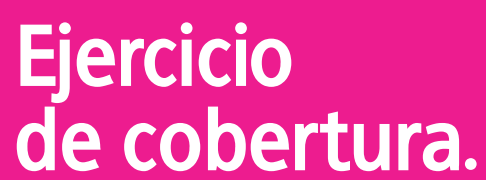

Una herramienta de evaluación rápida para programas y servicios de adolescente

\section{El Ejercicio de Cobertura}

El Ejercicio de Cobertura - EC- es una herramienta de monitoreo y evaluación sencilla, rápida y de bajo costo que permite identificar y caracterizar a los usuarios/as de un determinado servicio o grupo de proveedores/as de servicios u organizaciones que operan dentro de un área geográfica particular. Esta herramienta beneficia a las organizaciones individuales porque permite entender a quién están llegando los servicios. Permite además identificar problemas comunes entre organizaciones y que las instituciones los compartan y los discutan; comprender el acceso diferenciado a los servicios en un área y conocer a que población están llegando en la zona y si hay algunas que no están siendo alcanzadas; identificar vacíos programáticos y/o modificar el alcance de los programas.

A la fecha se han llevado a cabo Ejercicios de Cobertura en Etiopía, Burkina Faso, Guinea Bissau y Mauritania con el apoyo de USAID, PACT, UNFPA, entre otros. En Guatemala, el EC se realizó con apoyo del Consejo de Población. Su objetivo consistió en contar con información específica de la población adolescente (10 - 19 años) y joven (20 - 24 años) que asiste a actividades preventivas y asistenciales, para determinar si los servicios que se están ofertando están llegando a la población meta y si los mismos servicios son apropiados para quienes los están recibiendo.

La metodología para llevar a cabo este ejercicio incluyó:

1) Selección de socios. Se logró la participación voluntaria de ocho organizaciones que ofrecen las siguientes estrategias de atención a los adolescentes: charlas, atención clínica, educación de pares, embarazo simulado/bebés electrónicos y capacitaciones.

2) Sensibilización y capacitación del personal. Se realizaron talleres de capacitación en el uso de los formatos de recolección que sirvieron para registrar la información.

3) Recolección de datos. La recolección de datos estuvo a cargo de las organizaciones. Se realizó de junio a diciembre del 2007. Para ello se tomaron registro de los y las adolescentes y jóvenes (usuarios/as comprendidos/as entre las edades de 10 a 24 años) que asisten a diversas actividades organizadas en la comunidad o centros educativo.

4) Ingreso y análisis de los datos.

5) Divulgación de los datos y reevaluación de programas y políticas.

\section{Mayor cobertura en área urbana y en población ladina}

El 92.2\% de los jóvenes fueron atendidos en áreas urbanas del país. Solamente el $7.8 \%$ de los jóvenes se benefició de algún servicio o bien participó en actividades realizadas en áreas rurales del país. Las organizaciones con mayor cobertura en área rural alcanzan porcentajes del $100 \%$, $26.4 \%$ y $22.6 \%$.

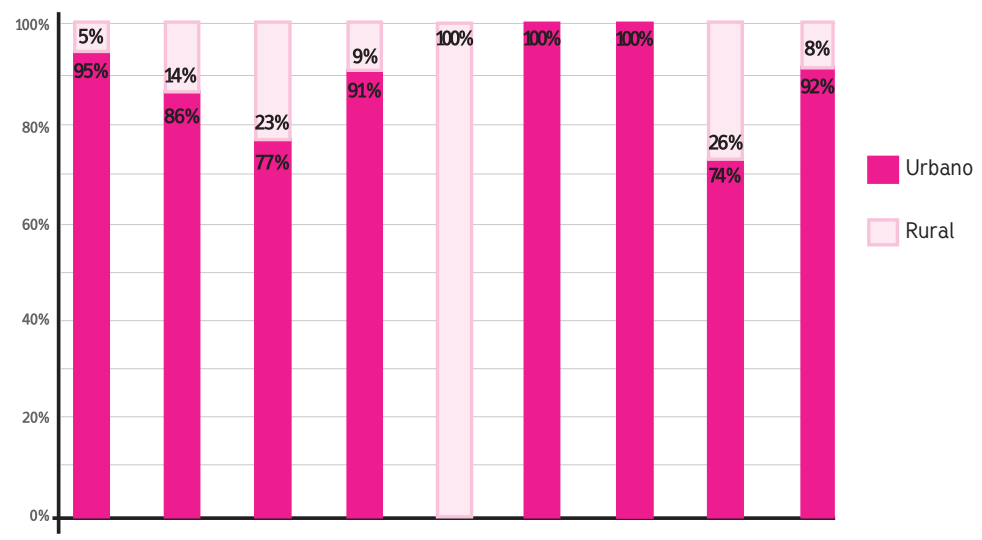


El $63.3 \%$ de los beneficiarios atendidos se autodefinen como ladinos, comparado con un $35.2 \%$ que se autodefinió como maya. Tomando como referencia el porcentaje de población indígena por departamento calculado a partir del censo de población del 2002 resulta que el porcentaje de cobertura de beneficiarios indígenas es casi igual que el porcentaje de población indígena en departamentos mayoritariamente indígenas como Totonicapán y Quiché. Existe sin embargo una brecha de cobertura de población indígena en algunos departamentos como Alta Verapaz, Huehuetenango y Sacatepéquez.

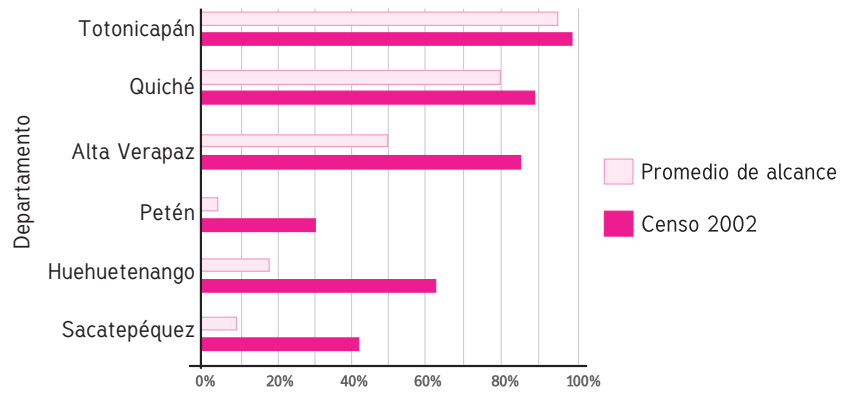

Beneficiarios principales: hombres y mujeres de 15 a 19 años, solteros y sin hijos

El $53.5 \%$ de los beneficiarios son mujeres y el $46.5 \%$ hombres. De acuerdo a su naturaleza, hay organizaciones que atienden solamente o mayoritariamente a mujeres. La mayoría, $54.3 \%$, de los beneficiarios y beneficiarias está comprendida en el rango de edad de 15 a 19 años. El $90.5 \%$ de los beneficiarios son solteros y el $91.6 \%$ reporta no tener hijos.

\section{Estudiantes miembros de hogares formados por ambos padres}

Según cifras del Ministerio de Educación, la tasa bruta de escolaridad para el ciclo básico es de 60.5. La tasa bruta de cobertura indica cuantos adolescentes, sin importar su edad, están asistiendo al ciclo básico, en relación a la población de 13 a 15 años del lugar. La tasa neta indica cuantos adolescentes en edad escolar están asistiendo al ciclo básico, en relación a la población de 13 a 15 años del lugar. Para el ciclo diversificado, esta tasa equivale a 20.7. Comparativamente, el EC revela que las organizaciones están Ilegando principalmente a jóvenes que están dentro del sistema educativo. El $85.0 \%$ de los niños y jóvenes atendidos por las organizaciones que participaron el EC están estudiando. Aproximadamente 9 de cada diez jóvenes hombres están estudiando, comparado con ocho de cada diez mujeres. 